CTP-TAMU-15/99, SINP-TNP/99-6

hep-th/9904129

\title{
Non-Threshold (F, Dp) Bound States
}

\author{
J. X. Lu ${ }^{1}$ and Shibaji Roy ${ }^{2}$ \\ ${ }^{1}$ Center for Theoretical Physics, Texas AEM University, College Station, TX 77843 \\ E-mail: jxlu@rainbow.physics.tamu.edu \\ ${ }^{2}$ Saha Institute of Nuclear Physics, 1/AF Bidhannagar, Calcutta 700 064, India \\ E-mail: roy@tnp.saha.ernet.in
}

\begin{abstract}
In the previous paper hep-th/9904112, we argued that there exist BPS bound states of Dp branes carrying certain units of quantized constant electric field for every $p$ (with $1 \leq p \leq 8$ ). Each of these bound states preserves one half of the spacetime supersymmetries. In this paper, we construct these bound state configurations explicitly for $2 \leq p \leq 7$ from Schwarz's $(m, n)$ string or (F, D1) bound state in type IIB string theory by T-dualities along the transverse directions. We calculate the charge per $(2 \pi)^{p-1} \alpha^{(p-1) / 2}$ of $(p-1)$-dimensional area for $\mathrm{F}$-strings in $(\mathrm{F}, \mathrm{Dp})$ and the tension for each of these bound states. The results agree precisely with those obtained previously from the worldvolume study. We study the decoupling limit for the (F, D3) bound state and find that Maldacena's $A d S_{5} / C F T_{4}$ correspondence may hold true even with respect to this bound state but now with an effective string coupling rather than the usual string coupling. This coupling is quantized and can be independent of the usual string coupling in certain limit.
\end{abstract}




\section{INTRODUCTION}

In the absence of a complete formulation of $\mathrm{M} /$ string theory, BPS $p$-brane solutions from various supergravities, which are the low energy and/or weak-coupling limits of this intrinsically non-perturbative unified theory, are almost the only sources from which reliable non-perturbative information about this theory can be extracted. These solutions played vital role in every major development in the past and will continue to do so. BPS properties obtained in the low energy and weak-coupling limits remain to be valid non-perturbatively for the corresponding BPS states. These properties should also be independent of whether they are obtained from fields in bulk spacetime or from fields on the corresponding worldvolume. For this reason, BPS properties obtained from the supergravity $p$-brane solutions are often used to obtain information about fields living on the corresponding worldvolume and viceversa. For example, the mass per unit p-brane volume calculated from a spacetime BPS Dp-brane solution should correspond to the Dp-brane tension of the corresponding BornInfeld action. This is trivial for simple Dp-brane solutions. But it is quite non-trivial for complicated solutions such as BPS bound state solutions. For example, the tension formula of $(m, n)$-string bound state of Schwarz [1], with $m, n$ relatively prime integers, can be used to determine the corresponding D-string worldsheet $U(1)$ field strength, in the linear approximation, as $g m$ with $g$ the string coupling [2]. This in turn can be used to determine the point charge due to the ending of a fundamental string (for short, F-string) on a general Dp-brane.

After all, a $p$-brane configuration represents the field configuration created by the corresponding $p$-brane source just like a static electric field due to a point charge represents the field surrounding the point source. Static interactions should be the same whether we calculate from the fields or from the sources. As mentioned above, BPS properties should be the same no matter whether they are obtained from the field configuration or from the source. However, apart from these BPS properties, one must be careful in using them since in general the fields and the source are not independent and they actually interact with each 
other through the so-called backreaction except in the case where the so-called decoupling limit is taken. In this limit, the modes propagating on the worldvolume will decouple from the modes propagating in the bulk spacetime even though these spacetime modes may have their origins from the worldvolume. So we expect that in the decoupling limit either the worldvolume fields or the bulk fields can be used to describe the same physics. Recently proposed $A d S / C F T$ correspondence by Maldacena [3] is one such example.

So BPS $p$-brane solutions can provide not only the non-perturbative information but also a basis for new types of strong-weak dualities. These are certainly important for us to seek the eventual formulation of $\mathrm{M} /$ string theory, along with the powerful D-brane picture of Polchinski [4.

In this paper, we will construct new spacetime solutions for BPS bound states of Dpbranes carrying certain units of the quantized worldvolume constant electric field strength. We argued in our previous paper [5] that such BPS bound states should exist for $1 \leq p \leq 8$ in both type IIA (when $p$ is even) and type IIB (when $p$ is odd) theoriest The existence of these bound states was also discussed in [6] but in a different approach of mixed boundary conditions. The non-vanishing $U(1)$ field strength on the worldvolume indicates that such a bound state carries information about F-strings ending on the Dp-brane, yet preserving one half of the spacetime supersymmetries. These Dp-brane bound states should be identified with the so-called (F, Dp) bound states which can be obtained from the $(m, n)$-string of Schwarz or $(F, D 1)$ bound state in type IIB string theory by T-dualities along the transverse directions. Here integers $m, n$ are relatively prime. This is precisely the method which we will use here to construct these solutions which was also mentioned in [7,9]. The $p=3,4,6$ configurations for (F, Dp) have been given in [7-9], respectively. Other non-threshold bound states for one $p^{\prime}$-brane within another p-brane with $p^{\prime}<p$ in $\mathrm{M}$ and Type II string theories have been discussed in $[7,10]$.

\footnotetext{
${ }^{1}$ In this paper, we specialize in cases of $2 \leq p \leq 7$.
} 
Our worldvolume picture of these Dp-brane bound states indicates clearly that the notation ' $\mathrm{F}$ ' in (F, Dp) represents actually an infinite number of parallel NS-strings. Precisely, we have one NS-string per $(2 \pi)^{p-1} \alpha^{\prime(p-1) / 2}$ area over a $(p-1)$-dimensional plane which is perpendicular to this string. Each NS-string is $m$ F-strings if the quantized worldvolume constant field strength $F_{01}=g m$, with $g$ the string coupling constant (where the field lines are chosen along $x^{1}$ axis). These were obtained in [5] by noting that the (F, D1) worldvolume action can be obtained from a (F, Dp), for $p \geq 2$, worldvolume action by T-dualities. By this, we also determined the tensions for (F, Dp) bound states in [5] which clearly indicates that $(\mathrm{F}, \mathrm{Dp})$ is a non-threshold bound state. We will show in this paper from the corresponding spacetime solutions that indeed these are all true.

Since we have constructed in this paper the space-time configurations for all the (F, Dp) bound states in type IIA and IIB theories, it would be natural to look at how they affect the $A d S / C F T$ correspondences conjectured by Maldacena. By examining the (F, D3) configuration carefully, we find that the decoupling limit in this case does not automatically imply the space-time geometry to be $A d S_{5} \times S^{5}$ as happens in the case of Maldacena, i.e., for simple D3-branes. We also find that the string coupling constant in this case is replaced by a finite but a smaller effective string coupling constant. However, we observed that by a suitable rescaling of the coordinates the $A d S_{5} / C F T_{4}$ correspondence may still hold true, but now with respect to (F, D3) bound state rather than the simple D3-branes. The effective string coupling constant in the near horizon region is quantized in terms of the integer $n$, the 5 -form flux and the integer $m$, the number of $\mathrm{F}$ strings per $(2 \pi)^{2} \alpha^{\prime}$ area over the two dimensional plane perpendicular to the $\mathrm{F}$ strings, where $m, n$ are relatively prime integers. This coupling can be independent of the usual string coupling at spatial infinity in the limit $m \gg n$.

This paper is organized as follows. In the following section, we discuss in some detail the so-called vertical and diagonal (or double) dimensional reductions/oxidations in type IIA and type IIB supergravities which are the basic methods we use to construct the (F, Dp) configurations. In section 3, we first give a detail construction of (F, D2) as an illustrating 
example. We then list results for each of $(\mathrm{F}, \mathrm{Dp})$ for $3 \leq p \leq 7$. We also present the (W, Dp) (with $\mathrm{W}$ representing the waves) solutions for $0 \leq p \leq 6$ from these newly found (F, Dp) configurations by T-dualities along the direction of F-strings. In section 4, we calculate the charge per unit $(p-1)$-dimensional area for the F-strings and show that we indeed have $m$ F-strings per $(2 \pi)^{p-1} \alpha^{(p-1) / 2}$ of $(p-1)$-dimensional area for these bound states. We also calculate the mass per unit p-brane volume for these solutions and show that the corresponding tensions agree precisely with what we obtained in [5] based on the worldvolume study. In section 5, we study the decoupling limit for the (F, D3) bound state. Finally, we conclude this paper in section 6.

\section{DIAGONAL AND VERTICAL DIMENSIONAL REDUCTIONS/OXIDATIONS}

It is well-known that type IIA and type IIB superstring theories compactified on a circle are equivalent by T-duality i.e. order by order in perturbation theory. More precisely, IIA and IIB theories are interchanged by the T-duality transformation $R \rightarrow 1 / R$, with $R$ the compactified radius, along with an interchange of momentum or KK modes with the winding modes. However, this equivalence does not extend to the respective $S^{1}$-compactified supergravity theories due to the absence of the string winding modes. But this will not have any effect on the massless modes in $D=9$. Therefore, the $D=9, N=2$ supergravity obtained from type IIA supergravity by the dimensional reduction must be equivalent by Tduality to that obtained from type IIB supergravity. This also follows from supersymmetry because $D=9, N=2$ supergravity is unique up to field redefinitions. Hence, the T-duality acting on the $D=9$ massless fields must map fields in IIA basis to those in IIB basis. This feature, as we will see, is precisely what we need to obtain a BPS solution in type IIA theory from a BPS solution in type IIB theory and vice versa by a T-duality transformation.

It is well-known that type II theories contain the following simple BPS states:2]:

\footnotetext{
${ }^{2}$ By simple, we mean an object whose charge is associated with only one antisymmetric tensor field
} 


$\begin{array}{llllllllllll}\text { Type IIA } & \text { W } & \text { F } & \text { NS5 } & \text { KK } & & \text { D0 } & \text { D2 } & \text { D4 } & \text { D6 } & \text { D8 } \\ \text { Type IIB } & \text { W } & \text { F } & \text { NS5 } & \text { KK } & & \text { D(-1) } & \text { D1 } & \text { D3 } & \text { D5 } & \text { D7 }\end{array}$

where W, F, NS5 and KK denote waves, fundamental strings, NS fivebranes, and KK monopoles, respectively, and they are associated with the NSNS fields. Also, Dp $(-1 \leq$ $p \leq 8)$ are the so-called D-branes and they are associated with the so-called RR fields. The action of T-duality along parallel or transverse direction on the above objects present in type II theories is given in the following table.

\begin{tabular}{|c|c|c|}
\hline & Parallel & Transverse \\
\hline Dp & $\mathrm{D}(\mathrm{p}-1)$ & $\mathrm{D}(\mathrm{p}+1)$ \\
$\mathrm{F}$ & $\mathrm{W}$ & $\mathrm{F}$ \\
$\mathrm{W}$ & $\mathrm{F}$ & $\mathrm{W}$ \\
NS5 & NS5 & KK \\
KK & KK & NS5 \\
\hline
\end{tabular}

The T-duality may be performed along either one of the worldvolume directions or one of the transverse directions (for KK monopole, the transverse direction is taken to be the nut direction).

It is clear from the above table that if we start from a (F, D1) bound state in the type IIB theory, we will have (F, D2) bound state in type IIA theory by T-dualizing (F, D1) along one of its transverse directions. Then we can have (F, D3) bound state in the type IIB theory by T-dualizing (F, D2) along one of its transverse directions. Repeating this procedure, we can have (F, D4) from (F, D3), (F, D5) from (F, D4), (F, D6) from (F, D5) and finally (F, D7) from (F, D6). We cannot obtain (F, D8) from (F, D7) by this procedure.

in type II theories. We have not included here the D9 branes, i.e., the so-called spacetime-filling branes [11]. 
We will explain possible reasons behind this at the end of this section. If we T-dualize the above bound states along the longitudinal direction of $\mathrm{F}$ in $(\mathrm{F}, \mathrm{Dp})$ for $1 \leq p \leq 7$, we have (W, Dp) bound states for $0 \leq p \leq 6$ with $\mathrm{W}$ representing the waves.

The question now is how to implement the above T-duality at the level of solutions of the IIA and IIB supergravities. As discussed above, the $S^{1}$ compactified IIA and IIB supergravities are not equivalent by T-duality except for the massless sector of $D=9, N=2$ supergravity. This implies that T-duality can be implemented in obtaining solutions in one supergravity from the known solutions each with one isometry along the would-be compactified direction in the other supergravity. This is exactly the rationale behind the procedure of the so-called vertical dimensional reduction and diagonal (or double) dimensional oxidation or the diagonal reduction and vertical oxidation, depending on whether the T-duality acts along a transverse direction or a worldvolume direction.

In this paper, we choose a convention such that $H_{n}$ denotes an $n$-form field strength in type IIB supergravity or in $D=9, N=2$ supergravity in terms of the type IIB basis and similarly $F_{n}$ denotes an $n$-form in type IIA supergravity or in $D=9, N=2$ supergravity in terms of the type IIA basis. If an $n$-form is reduced to an $(n-1)$-form from $D=10$ to $D=9$, we denote the resulting $(n-1)$-from with a superscript ' 1 ' indicating that it is obtained by one-step reduction. For example, if the reduction is along one of the indices of $H_{n}$, we have $H_{n} \rightarrow H_{n-1}^{(1)} \wedge d z$.

In the case of T-duality along a transverse direction of a BPS $p$-brane solution $(p \leq 6)$ as discussed in 12, 13, we first use the "no-force" condition for the $p$-brane configuration to obtain a multi-center solution from a single center one by placing many $p$-branes parallel to each other. Without loss of generality and to be specific, let us assume that this $p$-brane is in type IIB supergravity. The multi-center solution can be obtained by the following replacement in the corresponding Harmonic function. For example, if a single-center Harmonic function $H=1+Q_{p} / r^{7-p}$ with $r^{2}=\left(y^{1}\right)^{2}+\left(y^{2}\right)^{2}+\cdots+\left(y^{9-p}\right)^{2}$, then the multi-center Harmonic function will be given as, 


$$
H=1+\sum_{n} \frac{Q_{p}^{(n)}}{\left|\vec{y}-\vec{y}_{n}\right|^{7-p}}
$$

In order to generate one isometry along the T-dual direction, say, $y^{9-p}$-direction, as pointed out in [13], a continuous stack of $p$-branes with uniform charge density should be placed along the coordinate $z=y^{9-p}$ such that the summation in Eq. (2.2) can be replaced by an integration?. For the purpose of this paper, let us take a close look at this continuum limit. We first take $\vec{y}_{n}=2 \pi n a \hat{z}$ in Eq. (2.2), with $n \in Z, \hat{z}$ the unit vector along $y^{9-p}$-direction and $a=\sqrt{\alpha^{\prime}}$ where $\alpha^{\prime}$ is the string constant. If we allow the range of the summation in Eq. (2.2) to be $-\infty<n<\infty$ and take all charges to be equal, i.e., $Q_{p}^{(n)}=Q_{p}$, then the multi-center solution is an infinite periodic array of $p$-branes. The solution would be a stringy one if we take $\alpha^{\prime} \rightarrow 0$. Under this limit, the summation in Eq. (2.2) can also be replaced by an integration, i.e.,

$$
Q_{p} \sum_{n=-\infty}^{\infty} \frac{1}{|\vec{y}-2 \pi n a \hat{z}|^{7-p}} \rightarrow \frac{Q_{p}}{2 \pi a} \int_{-\infty}^{\infty} \frac{d z}{\left(r^{2}+z^{2}\right)^{(7-p) / 2}}=\frac{\tilde{Q}_{p}}{r^{6-p}}
$$

where $\tilde{Q}_{p}=c_{p} Q_{p}$ with constant $c_{p}=Q_{p+1} / Q_{p}$. The explicit expressions for $Q_{p+1}$ and $Q_{p}$ are given in the next section. In the above, we have now $r^{2}=\left(y^{1}\right)^{2}+\cdots+\left(y^{8-p}\right)^{2}$. In other words, one isometry is generated along the T-dual direction by the infinite periodic array of $p$-branes. Once this solution is known, a BPS $p$-brane solution in $D=9$ can be obtained from it. The Einstein metric for $D=9 p$-brane can be obtained from the corresponding metric of $D=10 p$-brane as (see, for example, the appendix of [14])

$$
d s_{10}^{2}(\text { type IIB })=e^{-\varphi_{B} /(2 \sqrt{7})} d s_{9}^{2}+e^{\sqrt{7} \varphi_{B} / 2} d z^{2},
$$

where $\varphi_{B}$ is a dilatonic scalar originating from the dimensional reduction. Since we know $d s_{10}^{2}$ (type IIB) and it also has one isometry along $z$-direction, we can read from the above the new field $\varphi_{B}$ and therefore the $D=9$ Einstein metric $d s_{9}^{2}$. The $D=10$ dilaton $\phi_{B}$ remains the same as the one for the solution of the infinite periodic array of $p$-branes in

\footnotetext{
${ }^{3}$ This seems to work also for non-BPS solutions as discussed in 13].
} 
$D=10$. The rule for reducing an $n$-form field strength $H_{n}$ is as follows: if it carries an electric-like charge, it will remain the same in $D=9$ whereas it will reduce to an $(n-1)$ form, i.e., $H_{n} \rightarrow H_{n-1}^{(1)} \wedge d z$, if it carries a magnetic charge. So we now obtain a $D=9$ BPS $p$-brane solution from a $D=10$ BPS $p$-brane solution by the so-called vertical dimensional reduction.

We expect that the T-dual of the above $p$-brane in type IIB gives a $(p+1)$-brane in type IIA. Let us first pretend that we know this $(p+1)$-brane solution in type IIA. We denote the brane coordinate $x^{p+1}=z$ (We choose the brane along $x^{1}, \cdots, x^{p+1}$-directions). All the fields for a static BPS $(p+1)$-brane are usually independent of the brane coordinates. So we have one isometry already along the $z$-direction. Thus a BPS $p$-brane solution in $D=9$ can be obtained simply by the so-called diagonal or double dimensional reduction [15] on the BPS $(p+1)$-brane solution of type IIA supergravity. The two Einstein metrics are now simply related to each other by

$$
d s_{10}^{2}(\text { type IIA })=e^{-\varphi_{A} /(2 \sqrt{7})} d s_{9}^{2}+e^{\sqrt{7} \varphi_{A} / 2} d z^{2},
$$

where $\varphi_{A}$ is another dilatonic scalar due to this dimensional reduction. The IIA dilaton $\phi_{A}$ remains unchanged. The $n$-form field strength $F_{n}$ is reduced according to the following: if it carries an electric charge, it will become an $(n-1)$-form in $D=9$, i.e., $F_{n} \rightarrow F_{n-1}^{(1)} \wedge d z$, whereas it will remain the same if it carries a magnetic charge, just the opposite to the case of vertical reduction. Here we do not really change anything. It is just the same whether we call the solution a $(p+1)$-brane in $D=10$ or a $p$-brane in $D=9$. In other words, if we know a $p$-brane solution in $D=9$, we should obtain a $(p+1)$-brane solution in $D=10$ right away and vice-versa. The process of obtaining a $(p+1)$-brane in $D=10$ from a $p$-brane in $D=9$ is called the diagonal oxidation.

The $p$-brane solution in $D=9$ obtained from a known type IIB $p$-brane solution is described in terms of fields in type IIB basis while in obtaining a $(p+1)$-brane in type IIA, we need a $p$-brane solution in $D=9$ described in terms of the fields in type IIA basis. So we need to map the fields in type IIB basis to fields in type IIA basis. This mapping is nothing 
but the T-duality transformation for the solution. This is also the T-duality transformation acting on the fields of $D=9, N=2$ supergravity discussed earlier. Then T-duality on field strengths in $D=9, N=2$ supergravity or the corresponding $p$-brane solutions is the identification $H_{n}=F_{n}$ with the understanding that if $H_{n}$ is defined with a Chern-Simons term so is $F_{n}$. The mapping for the $D=10$ dilaton $\phi$ and the dilatonic scalar $\varphi$ between the two versions of $D=9, N=2$ supergravity is

$$
\left(\begin{array}{c}
\phi_{A} \\
\varphi_{A}
\end{array}\right)=\left(\begin{array}{cc}
\frac{3}{4} & -\frac{\sqrt{7}}{4} \\
-\frac{\sqrt{7}}{4} & -\frac{3}{4}
\end{array}\right)\left(\begin{array}{c}
\phi_{B} \\
\varphi_{B}
\end{array}\right) .
$$

The above relations have been given or can be deduced, for example, from the appendix in [14] where the relations between the corresponding gauge potentials are also givenf.

Using the above relations, we can obtain a $p$-brane solution in $D=9$ in type IIA basis from a $p$-brane solution in type IIB basis. Then we obtain a BPS $(p+1)$-brane solution in type IIA by oxidizing this $p$-brane solution back to $D=10$ as described above.

If we reverse the above process, we then obtain a BPS $p$-brane solution in one theory from a known BPS $(p+1)$-brane solution in the other theory now by a T-duality along a world volume direction. The oxidation involved in this process is the so-called vertical one [13.

Now we conclude this section by explaining why we cannot obtain D8 brane solution in the massive type IIA supergravity from a D7 solution in the type IIB supergravity by a Tduality transformation along one of its transverse directions. First, it is not clear even now whether the massive type IIA supergravity has its origin in any of the known superstring theories or even in M-theory (though we believe so). So it is not clear that there exists a

\footnotetext{
${ }^{4}$ The precise relations between various fields in two different bases of the nine dimensional theory are given in a recent paper [16]. We would like to thank Chris Pope for letting us know their results prior to publication. These relations are also given in [17 but in a different conventions than we use here.
} 
theory from which the massive type IIA supergravity originates and is T-dual to the type IIB superstring theory. Even if we have such a theory, the massless fields in $D=9$ from the $S^{1}$-compactified massive type IIA supergravity cannot describe the 8 -brane in $D=9$. Therefore, we do not expect that the D8-brane solution is related to a D7 brane solution in the type IIB supergravity by a T-duality transformation?. Technically, as explained in [13], the vertical reduction does not seem to apply when $p=7$. By the same token, we cannot obtain (F, D8) solution from (F, D7) solution by a T-duality transformation.

\section{III. $\left(F, D_{P}\right)$ BOUND STATES}

In this section, we will construct explicitly the $(\mathrm{F}, \mathrm{Dp})$ bound state configurations for $2 \leq p \leq 7$ from the known (F, D1) bound state configuration of Schwarz [1] by a T-duality transformation along one of the transverse directions described in the previous section. We will give a detail construction for the case of (F, D2) as an example and present only the results for the rest. Once we have $(\mathrm{F}, \mathrm{Dp})$ configurations for $1 \leq p \leq 7$, we will also present the results for $(\mathrm{W}, \mathrm{Dp})$ for $0 \leq p \leq 6$, i.e., a $\mathrm{Dp}$ brane carrying waves in it, by a T-duality along the longitudinal direction of F-strings. All these configurations preserve one half of the spacetime supersymmetries.

The field configurations of the $(m, n)$-string of Schwarz (or (F, D1) bound state), with $m, n$ relatively prime integers, in type IIB theory [1] are given in terms of our notations as: the Einstein metric,

$$
d s^{2}(\text { type IIB })=H^{-3 / 4}\left[-\left(d x^{0}\right)^{2}+\left(d x^{1}\right)^{2}\right]+H^{1 / 4} d y^{i} d y^{i}
$$

with $i=1, \cdots, 8$; the type IIB dilaton,

$$
e^{\phi_{B}}=e^{\phi_{B 0}} H^{\prime} H^{-1 / 2}
$$

\footnotetext{
${ }^{5}$ We merely mean this at the level of supergravity solutions. As we know, in string theory, D8 brane does seem to be related to D7 brane by a T-duality.
} 
the axion,

$$
\chi_{B}=\frac{m n(H-1)+\chi_{B 0} \Delta_{(m, n)} e^{\phi_{B 0}}}{n^{2} H+\left(m-\chi_{B 0} n\right)^{2} e^{2 \phi_{B 0}}},
$$

and the NSNS 3-form field strength $H_{3}$ (NSNS) and RR 3-form $H_{3}$ (RR),

$$
\begin{aligned}
H_{3}(\mathrm{NSNS}) & =-\Delta_{(m, n)}^{-1 / 2} e^{\phi_{B 0}}\left(m-\chi_{B 0} n\right) d H^{-1} \wedge d x^{0} \wedge d x^{1}, \\
H_{3}(\mathrm{RR}) & =\Delta_{(m, n)}^{-1 / 2}\left[\chi_{B 0}\left(m-\chi_{B 0} n\right) e^{\phi_{B 0}}-n e^{-\phi_{B 0}}\right] d H^{-1} \wedge d x^{0} \wedge d x^{1} .
\end{aligned}
$$

In the above, $\phi_{B 0}$ and $\chi_{B 0}$ represent the asymptotic values of the type IIB dilaton and axion, respectively. The $\mathrm{SL}(2, \mathrm{Z})$ invariant $\Delta$-factor is

$$
\Delta_{(m, n)}=\left(m-\chi_{B 0} n\right)^{2} e^{\phi_{B 0}}+n^{2} e^{-\phi_{B 0}}
$$

and the $\mathrm{SL}(2, \mathrm{Z})$ invariant Harmonic function $H$ is

$$
H=1+\frac{Q_{1}}{r^{6}}
$$

where the radial distance $r^{2}=\left(y^{1}\right)^{2}+\left(y^{2}\right)^{2}+\cdots+\left(y^{8}\right)^{2}$ and the quantized central charge $Q_{1}$ is

$$
Q_{1}=2^{5} \pi^{2} \alpha^{\prime 3} \Delta_{(m, n)}^{1 / 2}
$$

with $\alpha^{\prime}$ the string constant. We have also introduced a second Harmonic function

$$
H^{\prime}=\frac{\left(m-\chi_{B 0} n\right)^{2} e^{\phi_{B 0}}+n^{2} H e^{-\phi_{B 0}}}{\Delta_{(m, n)}}
$$

which approaches unity as $r \rightarrow \infty$.

Before we move on to the constructions of (F, Dp) bound states, we fix a few conventions. The RR-charge of the Dp-brane in $(F, D p)$ is defined [18] as,

$$
e_{p}=\frac{1}{\sqrt{2} \kappa_{0}} \int_{S^{8-p}} e^{-a(p) \phi} * G_{p+2}
$$

for Noether "electric" charge, and

$$
g_{p}=\frac{1}{\sqrt{2} \kappa_{0}} \int_{S^{p+2}} G_{p+2}
$$


for topological or magnetic-like charge. In the above $\sqrt{2} \kappa_{0}=(2 \pi)^{7 / 2} \alpha^{\prime 2}$. For a Noether charge, the integration is over an asymptotic $(8-p)$-sphere surrounding the Dp-brane while for a magnetic-like charge, the integration is over an asymptotic $(p+2)$-sphere surrounding the Dp-brane. Also, $G_{p+2}=H_{p+2}$ for type IIB theory while $G_{p+2}=F_{p+2}$ for type IIA theory. The constant $a(p)=(p-3) / 2$ for Dp-branes in both type IIA and type IIB theories. The above definitions are also valid for NSNS branes, i.e., NSNS strings and fivebranes, but with $a(p)=-(p-3) / 2$. However, the charge associated with the F-strings in the (F, Dp) bound states cannot be calculated using the above simple formula. We will discuss this in the next section. In this paper, * always denotes the Hodge dual. $\epsilon_{n}$ denotes the volume form on an $n$-sphere where the volume of a unit $n$-sphere is

$$
\Omega_{n}=\frac{2 \pi^{(n+1) / 2}}{\Gamma((n+1) / 2)},
$$

and the unit charge for a Dp-brane is given as,

$$
Q_{0}^{p} \equiv(2 \pi)^{(7-2 p) / 2} \alpha^{\prime(3-p) / 2}
$$

(F, D2) Bound State: Now in order to construct this bound state we first need to construct, as described in the previous section, the solution corresponding to an infinite periodic array of $(m, n)$-strings along $z=y^{8}$ axis. The Harmonic function in that case would be given as,

$$
H=1+Q_{1} \sum_{n=-\infty}^{\infty} \frac{1}{|\vec{y}-2 \pi n a \hat{z}|^{6}}
$$

where $\hat{z}$ denotes the unit vector and $a=\sqrt{\alpha^{\prime}}$. In the limit $\alpha^{\prime} \rightarrow 0$, the summation in the above Harmonic function can be replaced by an integration, i.e.,

$$
\sum_{n=-\infty}^{\infty} \rightarrow \int_{-\infty}^{\infty} \frac{d z}{2 \pi a}
$$

Note that writing $\vec{y}=\tilde{\vec{y}}+z \hat{z}$ and so, $|\vec{y}-2 \pi n a \hat{z}|^{6}=\left[\tilde{r}^{2}+(z-2 \pi n a)^{2}\right]^{3}$, with $\tilde{r}^{2}=$ $\left(y^{1}\right)^{2}+\cdots+\left(y^{7}\right)^{2}$, we have,

$$
\sum_{n=-\infty}^{\infty} \frac{1}{|\vec{y}-2 \pi n a \hat{z}|^{6}} \rightarrow \int_{-\infty}^{\infty} \frac{d z}{2 \pi a} \frac{1}{\left(\tilde{r}^{2}+z^{2}\right)^{3}}=\frac{3}{16 a} \frac{1}{\tilde{r}^{5}}
$$


So the new Harmonic function describing the infinite array of $(m, n)$-strings along the $z$-direction is

$$
H=1+\frac{Q_{2}}{r^{5}}
$$

where we have dropped the 'tilde' above the radial distance and will continue to do so from now on. The central charge $Q_{2}$ in $(3.16)$ is

$$
Q_{2}=\frac{3 Q_{1}}{16 \alpha^{\prime 1 / 2}}=6 \pi^{2} \alpha^{15 / 2} \Delta_{(m, n)}^{1 / 2},
$$

The Harmonic function is now independent of the $z$-coordinate. In other words, the Einstein metric (Eq. (3.1)) expressed in terms of this Harmonic function possesses one additional isometry along the $z$-direction. If we now compactify the metric along $z$-direction, Eq. (2.4) will give

$$
e^{\sqrt{7} \varphi_{B} / 2}=H^{1 / 4}
$$

and the 9-d Einstein metric will be given as,

$$
d s_{9}^{2}=H^{-5 / 7}\left[-\left(d x^{0}\right)^{2}+\left(d x^{1}\right)^{2}\right]+H^{2 / 7} d y^{i} d y^{i}
$$

where $i=1,2, \cdots, 7$. For the 9 -dimensional solution, the type IIB dilaton, axion and NSNS and RR 3-forms remain unchanged with the replacement of the old Harmonic function by the new one. We have therefore obtained $(m, n)$-string bound state in $D=9$ in the type IIB basis.

We now express all the relevant fields in type IIA basis. The $d s_{9}^{2}$ remains unchanged. $F_{3}=H_{3}(\mathrm{NSNS}), F_{3}^{(1)}=H_{3}(\mathrm{RR})$ and $F_{1}^{(1)}=d \chi$. The type IIA dilaton $\phi_{A}$ and the dilatonic scalar $\varphi_{A}$ can be obtained from $\phi_{B}$ and $\varphi_{B}$ through Eq. (2.6) as

$$
\begin{aligned}
e^{\phi_{A}} & =e^{3 \phi_{B 0} / 4} H^{\prime 3 / 4} H^{-1 / 2}, \\
e^{\sqrt{7} \varphi_{A}} & =e^{-7 \phi_{B 0} / 4} H^{\prime-7 / 4} H^{1 / 2},
\end{aligned}
$$

where the Harmonic function $H^{\prime}$ continues to be given by the expression (3.8) but in terms of the present Harmonic function $H$. 
Once we express $D=9$ (F, D1) configuration in the type IIA basis, we can read the (F, D2) configuration in type IIA theory using the diagonal oxidation described in the previous section. For example, the Einstein metric in $D=10$ for this configuration can be read from Eq. (2.5) with $d s_{9}^{2}, \phi_{A}$ and $\varphi_{A}$ given in (3.19) and (3.20). We here collect the complete results for the (F, D2) configuration for the metric,

$$
d s_{10}^{2}(\text { type IIA })=e^{\phi_{B 0} / 8} H^{\prime / 8} H^{1 / 4}\left[H^{-1}\left(-\left(d x^{0}\right)^{2}+\left(d x^{1}\right)^{2}\right)+e^{-\phi_{B 0}} H^{\prime-1}\left(d x^{2}\right)^{2}+d y^{i} d y^{i}\right]
$$

for the dilaton,

$$
e^{\phi_{A}}=e^{3 \phi_{B 0} / 4} H^{\prime 3 / 4} H^{-1 / 2}
$$

and for the remaining non-vanishing fields

$$
\begin{aligned}
& F_{2}=n \Delta_{(m, n)}^{-1} e^{-\phi_{B 0}}\left(m-\chi_{B 0} n\right) H^{\prime-2} d H \wedge d x^{2}, \\
& F_{3}=-\Delta_{(m, n)}^{-1 / 2} e^{\phi_{B 0}}\left(m-\chi_{B 0} n\right) d H^{-1} \wedge d x^{0} \wedge d x^{1}, \\
& F_{4}^{\prime}=n e^{-3 \phi_{B 0} / 8} H^{\prime-3 / 8} H^{1 / 4} \frac{\sqrt{2} \kappa_{0} Q_{0}^{2}}{\Omega_{5}} * \epsilon_{6} .
\end{aligned}
$$

In the above, $i=1,2, \cdots 7$, the Harmonic functions $H^{\prime}$ and $H$ are given by Eqs. (3.8) and (3.16), respectively.

(F, D3) Bound Statef: Once we obtain (F, D2), we can repeat the above process to obtain (F, D3) bound state configuration. The results are for the Einstein metric,

$$
\begin{aligned}
d s_{10}^{2}(\text { type IIB })= & e^{\phi_{B 0} / 4} H^{1 / 4} H^{1 / 4}\left[H^{-1}\left(-\left(d x^{0}\right)^{2}+\left(d x^{1}\right)^{2}\right)\right. \\
& \left.+e^{-\phi_{B 0}} H^{\prime-1}\left(\left(d x^{2}\right)^{2}+\left(d x^{3}\right)^{2}\right)+d y^{i} d y^{i}\right]
\end{aligned}
$$

\footnotetext{
${ }^{6}$ In type IIA theory, $F_{4}^{\prime}=d A_{3}+A_{1} \wedge F_{3}$

${ }^{7}$ This configuration with zero asymptotic values of $\phi_{B 0}$ and $\chi_{B 0}$ was also given in [7] for different purpose. Some non-threshold bound states of M theory, not considered here, were also discussed there.
} 
with $i=1,2, \cdots, 6$; for the type IIB dilaton,

$$
e^{\phi_{B}}=e^{\phi_{B 0} / 2} H^{1 / 2} H^{-1 / 2}
$$

and for the remaining non-vanishing fields,

$$
\begin{aligned}
H_{3}(\mathrm{NSNS}) & =-\Delta_{(m, n)}^{-1 / 2} e^{\phi_{B 0}}\left(m-\chi_{B 0} n\right) d H^{-1} \wedge d x^{0} \wedge d x^{1} \\
H_{3}(\mathrm{RR}) & =n \Delta_{(m, n)}^{-1} e^{-\phi_{B 0}}\left(m-\chi_{B 0} n\right) H^{\prime-2} d H \wedge d x^{2} \wedge d x^{3}, \\
H_{5} & =n \frac{\sqrt{2} \kappa_{0} Q_{0}^{3}}{\Omega_{5}}\left(* \epsilon_{5}+\epsilon_{5}\right) .
\end{aligned}
$$

In the above, the Harmonic function $H^{\prime}$ continues to be given by Eq. (3.8) but the Harmonic function $H$ is now

$$
H=1+\frac{Q_{3}}{r^{4}}
$$

where $Q_{3}=\Delta_{(m, n)}^{1 / 2} \sqrt{2} \kappa_{0} Q_{0}^{3} /\left(4 \Omega_{5}\right)$. All the other quantities have already been defined.

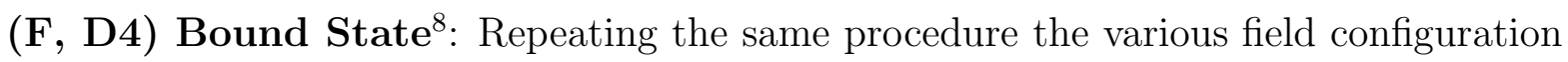
for this solution are: the metric,

$$
\begin{aligned}
d s_{10}^{2}(\text { type IIA })= & e^{3 \phi_{B 0} / 8} H^{\prime 3 / 8} H^{1 / 4}\left[H^{-1}\left(-\left(d x^{0}\right)^{2}+\left(d x^{1}\right)^{2}\right)\right. \\
& \left.+e^{-\phi_{B 0}} H^{\prime-1}\left(\left(d x^{2}\right)^{2}+\left(d x^{3}\right)^{2}+\left(d x^{4}\right)^{2}\right)+d y^{i} d y^{i}\right],
\end{aligned}
$$

with $i=1,2, \cdots, 5$; the type IIA dilaton,

$$
e^{\phi_{A}}=e^{\phi_{B 0} / 4} H^{1 / 4} H^{-1 / 2}
$$

and the remaining non-vanishing fields,

$$
\begin{aligned}
& F_{3}=-\Delta_{(m, n)}^{-1 / 2} e^{\phi_{B 0}}\left(m-\chi_{B 0} n\right) d H^{-1} \wedge d x^{0} \wedge d x^{1} \\
& F_{4}^{\prime}=n \frac{\sqrt{2} \kappa_{0} Q_{0}^{4}}{\Omega_{4}} \epsilon_{4}+n \Delta_{(m, n)}^{-1} e^{-\phi_{B 0}}\left(m-\chi_{B 0} n\right) H^{\prime-2} d H \wedge d x^{2} \wedge d x^{3} \wedge d x^{4}
\end{aligned}
$$

\footnotetext{
${ }^{8}$ The classical configuration of this bound state was also given in [8], obtained by dimensional reductions from the $(\mathrm{M} 2, \mathrm{M} 5)$ bound state in $\mathrm{D}=11$.
} 
In the above, the Harmonic function $H^{\prime}$ is as given by Eq. (3.8) but the Harmonic function $H$ is now

$$
H=1+\frac{Q_{4}}{r^{3}}
$$

where $Q_{4}=\Delta_{(m, n)}^{1 / 2} \sqrt{2} \kappa_{0} Q_{0}^{4} /\left(3 \Omega_{4}\right)$.

(F, D5) Bound State: The field configurations for this bound state are: the Einstein metric,

$$
\begin{aligned}
d s_{10}^{2}(\text { type IIB })= & e^{\phi_{B 0} / 2} H^{1 / 2} H^{1 / 4}\left[H^{-1}\left(-\left(d x^{0}\right)^{2}+\left(d x^{1}\right)^{2}\right)\right. \\
& \left.+e^{-\phi_{B 0}} H^{\prime-1}\left(\left(d x^{2}\right)^{2}+\left(d x^{3}\right)^{2}+\left(d x^{4}\right)^{2}+\left(d x^{5}\right)^{2}\right)+d y^{i} d y^{i}\right],
\end{aligned}
$$

with $i=1,2,3,4$; the type IIB dilaton,

$$
e^{\phi_{B}}=H^{-1 / 2}
$$

and the remaining non-vanishing fields,

$$
\begin{aligned}
H_{3}(\mathrm{NSNS}) & =-\Delta_{(m, n)}^{-1 / 2} e^{\phi_{B 0}}\left(m-\chi_{B 0} n\right) d H^{-1} \wedge d x^{0} \wedge d x^{1}, \\
H_{3}(\mathrm{RR}) & =n \frac{\sqrt{2} \kappa_{0} Q_{0}^{5}}{\Omega_{3}} \epsilon_{3}, \\
H_{5} & =n \Delta_{(m, n)}^{-1} e^{-\phi_{B 0}}\left(m-\chi_{B 0} n\right) H^{\prime-2} d H \wedge d x^{2} \wedge d x^{3} \wedge d x^{4} \wedge d x^{5} .
\end{aligned}
$$

Again the Harmonic function $H^{\prime}$ remains the same as given in Eq. (3.8) but the Harmonic function $H$ is now

$$
H=1+\frac{Q_{5}}{r^{2}}
$$

where $Q_{5}=\Delta_{(m, n)}^{1 / 2} \sqrt{2} \kappa_{0} Q_{0}^{5} /\left(2 \Omega_{3}\right)$.

(F, D6) Bound Statef: In this case we have the Einstein metric,

$$
\begin{aligned}
d s_{10}^{2}(\text { type IIA })= & e^{5 \phi_{B 0} / 8} H^{\prime 5 / 8} H^{1 / 4}\left[H^{-1}\left(-\left(d x^{0}\right)^{2}+\left(d x^{1}\right)^{2}\right)\right. \\
& \left.+e^{-\phi_{B 0}} H^{\prime-1}\left(\left(d x^{2}\right)^{2}+\cdots+\left(d x^{6}\right)^{2}\right)+d y^{i} d y^{i}\right],
\end{aligned}
$$

\footnotetext{
${ }^{9}$ The classical configuration of this bound state was also given in $[9]$.
} 
with $i=1,2,3$; the type IIA dilaton,

$$
e^{\phi_{A}}=e^{-\phi_{B 0} / 4} H^{-1 / 4} H^{-1 / 2}
$$

and the remaining non-vanishing fields,

$$
\begin{aligned}
& F_{2}=n \frac{\sqrt{2} \kappa_{0} Q_{0}^{6}}{\Omega_{2}} \epsilon_{2}, \\
& F_{3}=-\Delta_{(m, n)}^{-1 / 2} e^{\phi_{B 0}}\left(m-\chi_{B 0} n\right) d H^{-1} \wedge d x^{0} \wedge d x^{1}, \\
& F_{4}^{\prime}=-n \Delta_{(m, n)}^{-1 / 2} e^{\phi_{B 0}}\left(m-\chi_{B 0} n\right) H^{-1} \frac{\sqrt{2} \kappa_{0} Q_{0}^{6}}{\Omega_{2}} d x^{0} \wedge d x^{1} \wedge \epsilon_{2},
\end{aligned}
$$

Once again the Harmonic function $H^{\prime}$ continues to be given by Eq. (3.8) but the Harmonic function $H$ is

$$
H=1+\frac{Q_{6}}{r}
$$

where $Q_{6}=\Delta_{(m, n)}^{1 / 2} \sqrt{2} \kappa_{0} Q_{0}^{6} / \Omega_{2}$.

(F, D7) Bound State: The various field configurations in this case are described by the following Einstein metric,

$$
\begin{aligned}
d s_{10}^{2}(\text { type IIB })= & e^{3 \phi_{B 0} / 4} H^{\prime 3 / 4} H^{1 / 4}\left[H^{-1}\left(-\left(d x^{0}\right)^{2}+\left(d x^{1}\right)^{2}\right)\right. \\
& \left.+e^{-\phi_{B 0}} H^{\prime-1}\left(\left(d x^{2}\right)^{2}+\left(d x^{3}\right)^{2}+\cdots+\left(d x^{7}\right)^{2}\right)+d y^{i} d y^{i}\right],
\end{aligned}
$$

with $i=1,2$; the type IIB dilaton,

$$
e^{\phi_{B}}=e^{-\phi_{B 0} / 2} H^{\prime-1 / 2} H^{-1 / 2}
$$

and the remaining non-vanishing fields,

$$
\begin{aligned}
d \chi & =n \frac{\sqrt{2} \kappa_{0} Q_{0}^{7}}{\Omega_{1}} \epsilon_{1}, \\
H_{3}(\mathrm{RR}) & =-n \Delta_{(m, n)}^{-1 / 2} e^{\phi_{B 0}}\left(m-\chi_{B 0} n\right) H^{-1} \frac{\sqrt{2} \kappa_{0} Q_{0}^{7}}{\Omega_{1}} d x^{0} \wedge d x^{1} \wedge \epsilon_{1}, \\
H_{3}(\mathrm{NSNS}) & =-\Delta_{(m, n)}^{-1 / 2} e^{\phi_{B 0}}\left(m-\chi_{B 0} n\right) d H^{-1} \wedge d x^{0} \wedge d x^{1} .
\end{aligned}
$$

Here the Harmonic function $H^{\prime}$ is again given by Eq. (3.8) but the Harmonic function $H$ is now 


$$
H=1-Q_{7} \ln r
$$

where $Q_{7}=\Delta_{(m, n)}^{1 / 2} \sqrt{2} \kappa_{0} Q_{0}^{7} / \Omega_{1}$.

A detail discussion of the meaning and the other properties of the above solutions will be given in the following section. We, however, continue this section to briefly indicate how to construct (W, Dp) bound states in type II theories from the (F, Dp) bound states already constructed.

So far, we have performed T-duality only along the transverse directions of the F-strings to obtain $(\mathrm{F}, \mathrm{Dp})$ bound states from the known (F, D1) bound state. But we note that following the prescription given in the previous section, we can also T-dualize the above (F, Dp) solutions for $1 \leq p \leq 7$ along $x^{1}$-direction of the F-strings. This will give (W, Dp) bound state solutions for $0 \leq p \leq 6$. We here present the Einstein metric, the dilaton and the Kaluza-Klein vector potential for each of these solutions. The remaining non-vanishing fields can be obtained easily from the corresponding ones of (F, Dp). For the (W, Dp) solutions we have the following Einstein metric,

$$
\begin{aligned}
d s_{10}^{2}= & e^{(p+1) \phi_{B 0} / 8} H^{\prime(p+1) / 8}\left[-H^{-1}\left(d x^{0}\right)^{2}+e^{-\phi_{B 0}} H^{\prime-1} H\left(d x^{1}+\mathcal{A}_{0} d x^{0}\right)^{2}\right. \\
& \left.+e^{-\phi_{B 0}} H^{\prime-1}\left(\left(d x^{2}\right)^{2}+\cdots+\left(d x^{p}\right)^{2}\right)+d y^{i} d y^{i}\right]
\end{aligned}
$$

with $i=1,2, \cdots,(9-p)$; the dilaton,

$$
e^{\phi}=e^{(3-p) \phi_{B 0} / 4} H^{\prime(3-p) / 4}
$$

and the Kaluza-Klein vector potential

$$
\mathcal{A}_{0}=-\Delta_{(m, n)}^{-1 / 2} e^{\phi_{B 0}}\left(m-\chi_{B 0} n\right) H^{-1}
$$

In the above, the Harmonic function $H$ is given by,

$$
H= \begin{cases}1+\frac{Q_{p+1}}{r^{6-p}}, & 0 \leq p \leq 5, \\ 1-Q_{7} \ln r, & p=6\end{cases}
$$

and the Harmonic function $H^{\prime}$ continues to be given by Eq. (3.8). All the other quantities are already given before. Note that for $m=0$ (also $\chi_{B 0}=0$ and for simplicity we also set 
$\phi_{B 0}=0[\square)$, we have $H^{\prime}=H$ and $\mathcal{A}_{0}=0$. Then the above solution represents an infinite periodic array of Dp-branes along the $x^{1}$-axis. While for $n=0, H^{\prime}=1$, and the above solution represents a gravitational wave propagating in $x^{1}$-direction with isometries along $\left(x^{1}, x^{2}, \cdots, x^{p}\right)$-directions. We will not discuss the (W, Dp) bound states any further in this paper and focus on (F, Dp) ones for the rest.

\section{PROPERTIES OF $\left(F, D_{P}\right)$ BOUND STATES}

The worldvolume picture for a (F, Dp) bound state described in [5] consists of constant electric field lines flowing along, say, $x^{1}$-axis in the Dp-brane worldvolume. This picture applies for each of our spacetime configurations of (F, Dp) given in the previous section. In order to see that, one can examine that for $m=0$ (with $\chi_{B 0}=0$ and $\phi_{B 0}=0$ for simplicity), the metric describes Dp-branes lying along $\left(x^{1}, x^{2}, \cdots, x^{p}\right)$-directions while for $n=0$ (hence $H^{\prime}=1$ ), it describes F-strings lying along the $x^{1}$-direction with isometries along $\left(x^{1}, x^{2}, \cdots, x^{p}\right)$-directions. So, this configuration indeed describes F-strings within the $\mathrm{D}$ branes in consistency with our worldvolume picture. In order to make such an identification more precise, we need to calculate the charges carried by the Dp-brane and by the F-strings, and also the mass per unit $p$-brane worldvolume, and compare them with what we obtained in [5] based on the worldvolume approach.

Let us first calculate the RR charge for the Dp-brane in a (F, Dp) bound state configuration given in the previous section. This can be done easily using either Eq.(3.9) or Eq.(3.10) depending on whether the charge is electric-like or magnetic-like with the corresponding explicit $(p+2)$-form field strength given in the previous section. We have $e_{p}=n Q_{0}^{p}$ for an electric-like $\mathrm{RR}$ charge or $g_{p}=n Q_{0}^{p}$ for a magnetic-like $\mathrm{RR}$ charge. The integer $n$ originates from that of the D-string in the (F, D1) bound state. So the RR charge is automatically quantized given the quantization of D-string charge, a well-known fact that the

\footnotetext{
${ }^{10}$ We will discuss this in the following section.
} 
charge quantization for one extended object will imply charge quantizations for the rest of the extended objects in string/M theory. This is consistent with our worldvolume result. Note that $Q_{0}^{p} Q_{0}^{6-p}=2 \pi$.

As hinted earlier, Eq. (3.9) cannot be applied simply to calculate the electric-like charge associated with the F-strings in $(\mathrm{F}, \mathrm{Dp})$. The reason is, as explained in [5], that the notation ' $\mathrm{F}$ ' in (F, Dp) means actually an infinite number of parallel strings in the bound state. As mentioned in [5], there is one NS-string (or equivalently $m$ F-strings) per $(2 \pi)^{p-1} \alpha^{(p-1) / 2}$ of $(p-1)$-dimensional area. We will show later in this section that this is indeed true. In $1+$ 3 dimensional electrostatics, we know that in order to use Gauss law to obtain the charge per unit length for a uniform line distribution of charge, we have to choose a cylinder (with the line charge at the center) as the Gauss surface rather than a 2-sphere as for a point charge. For the present case, we therefore should choose the integration in Eq. (3.9) over $R^{p-1} \times S^{8-p}$ rather than over an asymptotic $S^{7}$. We can therefore have charge per unit $(p-1)$-dimensional area for the F-strings.

In order to give meaningful calculations for this quantity and for the mass per unit Dp-brane worldvolume which will be used to determine the tension for a (F, Dp) bound state, we need a good asymptotic behavior for the metric such that the $(p-1)$-dimensional area and the worldvolume can be defined with respect to certain frame metric. This is also necessary for us to make comparisons with the results obtained in [5] based on the worldvolume analysis. Let us take a close look at each Einstein frame metric for those (F, Dp) bound state configurations. They, along with the corresponding dilatons, can actually be expressed in a unified way as

$$
\begin{aligned}
d s^{2}= & e^{(p-1) \phi_{B 0} / 8} H^{\prime(p-1) / 8} H^{1 / 4}\left[H^{-1}\left(-\left(d x^{0}\right)^{2}+\left(d x^{1}\right)^{2}\right)\right. \\
& \left.+e^{-\phi_{B 0}} H^{\prime-1}\left(\left(d x^{2}\right)^{2}+\cdots+\left(d x^{p}\right)^{2}\right)+d y^{i} d y^{i}\right]
\end{aligned}
$$

for the metric with $i=1,2, \cdots, 9-p$; and

$$
e^{\phi}=e^{(5-p) \phi_{B 0} / 4} H^{\prime(5-p) / 4} H^{-1 / 2} .
$$


for the dilaton. In the above, the Harmonic function $H$ is

$$
H=1+\frac{Q_{p}}{r^{7-p}},
$$

with

$$
Q_{p}=\frac{\Delta_{(m, n)}^{1 / 2} \sqrt{2} \kappa_{0} Q_{0}^{p}}{(7-p) \Omega_{8-p}}
$$

and the Harmonic function $H^{\prime}$ is given by Eq. (3.8).

Note first that the constants $\phi_{B 0}$ and $\chi_{B 0}$ appearing in all the above solutions are no longer the asymptotic values for the dilaton and the axion except for the original (F, D1) bound state configuration. Actually, the axion for $p=3,5,7$ has nothing to do with the constant $\chi_{B 0}$. In fact, $\chi_{B 0}$ is the asymptotic value of the $(p-1)$-form $\mathrm{RR}$ gauge potential in the corresponding $(\mathrm{F}, \mathrm{Dp})$ configuration. The asymptotic value of dilaton for every $p$ except for $p=5$ is related to the original $\phi_{B 0}$ but not equal. This can be understood from the fact that a T-duality transformation shifts the dilaton value [19]. From the dilaton expression for each of the above solutions, we can see that the asymptotic value of the dilaton is reduced by a quarter of $\phi_{B 0}$ for every T-duality transformation. For $p=5$, the asymptotic value vanishes. Second, even though we start with an Einstein metric for (F, D1) which is asymptotically Minkowski, none of the metrics derived by T-duality transformations remains to be so. Even worse, none of them remains asymptotically as a constant scaling factor times Minskowski metric. However, there exists a constraint on the asymptotic behavior of the Einstein metric for the (F, D1) bound state configuration which preserves not only the asymptotic behavior for each of the derived Einstein metrics but also the asymptotic value for the dilatons $\square$. This turns out to be the one which imposes the corresponding string metric rather than the Einstein metric to be asymptotically Minkowski. This constraint has been used in the literature and once again we see its significance. We will discuss possible reasons behind this and other related issues in a separate paper.

\footnotetext{
${ }^{11}$ This remains true also for the $(\mathrm{W}, \mathrm{Dp})$ bound states.
} 
Once we make such a choice for the asymptotic metric, Eq. (4.1) and (4.2) become

$$
\begin{aligned}
d s^{2}= & e^{-\phi_{B 0} / 2} H^{\prime(p-1) / 8} H^{1 / 4}\left[H^{-1}\left(-\left(d x^{0}\right)^{2}+\left(d x^{1}\right)^{2}\right)\right. \\
& \left.+H^{\prime-1}\left(\left(d x^{2}\right)^{2}+\cdots+\left(d x^{p}\right)^{2}\right)+d y^{i} d y^{i}\right]
\end{aligned}
$$

and

$$
e^{\phi}=e^{\phi_{B 0}} H^{\prime(5-p) / 4} H^{-1 / 2} .
$$

The Harmonic function $H$ is given by Eq. (4.3) but with $Q_{p} \rightarrow e^{3 \phi_{B 0} / 2} Q_{p}$ with $Q_{p}$ given in Eq. (4.4). The expressions (4.5) and (4.6) have the above mentioned properties. For example, the asymptotic value for the dilaton is always $\phi_{B 0}$, the one in the original (F, D1) bound state. In what follows, we simply use $\phi_{0}$ rather than $\phi_{B 0}$ as the asymptotic value for the dilaton. We give below the explicit form of NSNS 3-from field strength purpose of calculating the charge per unit $(p-1)$-dimensional area for F-strings which is

$$
G_{3}=-\Delta_{(m, n)}^{-1 / 2} e^{\phi_{0} / 2}\left(m-\chi_{B 0} n\right) d H^{-1} \wedge d x^{0} \wedge d x^{1} .
$$

As discussed above, the charge per unit $(p-1)$-dimensional area for the F-strings should be calculated as,

$$
e_{1}=\frac{1}{\sqrt{2} \kappa_{0}} \int_{R^{p-1} \times S^{8-p}}\left(e^{-\phi} * G_{3}+\cdots\right),
$$

where $\cdots$ indicates possible non-vanishing Chern-Simons terms. Actually, the Chern-Simons terms do contribute to this charge. The charge $e_{1}$ itself must be infinite since we have an infinite number of F-strings. But we expect that $e_{1} / A_{p-1}$ should be finite with $A_{p-1}=$ $\int d x^{2} \wedge \cdots \wedge d x^{p}$ the coordinate $(p-1)$-dimensional area. Indeed, we find

$$
\left|e_{1}\right| / A_{p-1}=m Q_{0}^{p},
$$

As mentioned in [5], we have one NSNS-string (or $m$ F-strings) per $(2 \pi)^{p-1} \alpha^{\prime(p-1) / 2}$ of $(p-1)$ dimensional area from the worldvolume point of view. So, in order to compare with our

\footnotetext{
${ }^{12} G_{3}=F_{3}$ in type IIA while $G_{3}=H_{3}$ (NSNS) in type IIB.
} 
interpretation for the F-strings along $x^{1}$-direction we must multiply the left side of the above equation with this $(p-1)$-dimensional area. Thus we obtain,

$$
(2 \pi)^{p-1} \alpha^{\prime(p-1) / 2} \frac{\left|e_{1}\right|}{A_{p-1}}=\sqrt{2} \kappa_{0} m T_{f},
$$

Now in order for the left hand side of Eq.(4.10) to represent the charge of F-strings per $(2 \pi)^{p-1} \alpha^{\prime(p-1) / 2}$ of $(p-1)$-dimensional area, we must replace the coordinate area $A_{p-1}$ by the one measured in string metric asymptotically. But, since we have chosen our string metric to be asymptotically flat, they are the same. Note also that according to our definition the charge $e_{p}$ of the $p$-brane is related to the corresponding tension $T_{p}$ as $e_{p}=\sqrt{2} \kappa_{0} T_{p}$ and therefore, Eq.(4.10) clearly states that there are indeed $m$ F strings in the (F, Dp) bound states per $(2 \pi)^{p-1} \alpha^{(p-1) / 2}$ of $(p-1)$-dimensional area, confirming our interpretation.

There is another way to obtain the above result which gives a direct link between the spacetime F-strings and the worldvolume electric flux lines. This will be important for our discussion of the $A d S_{5} / C F T_{4}$ correspondence given in section 5. The low-energy effective field theory on a Dp brane worldvolume contains a coupling,

$$
\frac{1}{T_{0}^{p} g} \int d^{p+1} \sigma B_{\mu \nu}\left(2 \pi \alpha^{\prime} F^{\mu \nu}\right)
$$

where $B_{2}$ is the pull-back of the NSNS 2-form potential on the worldvolume, $F$ is the worldvolume gauge field strength $\amalg$ ] $T_{0}^{p}=1 /\left[(2 \pi)^{p} \alpha^{(p+1) / 2}\right]$ is the $p$-brane tension units and $g=e^{\phi_{0}}$ is the string coupling. Because of this term the equation of motion takes the form (in terms of our conventions) ${ }^{14}$

$$
d\left(e^{-\phi} * G_{3}+\cdots\right)=\frac{2 \kappa_{0}^{2}}{g T_{f} T_{0}^{p}} * F \wedge \delta^{9-p},
$$

where $*$ in $* G_{3}$ denotes the Hodge dual in $D=10$ spacetime while the $*$ in $* F$ denotes the Hodge dual within the $(p+1)$-dimensional worldvolume. In the above, $\delta^{9-p}$ denotes

\footnotetext{
${ }^{13}$ Strictly speaking, we should use $\mathcal{F}=F-B_{2}$ instead.

${ }^{14}$ Here, we do not include an F-string source to the equation of motion since we are considering an infinite number of parallel F-strings in the bulk.
} 
a $(9-p)$-form delta function on the Dp brane worldvolume. We use this equation for the purpose of relating the F-string charge to the electric flux of gauge field on the worldvolume. (Note that a similar equation has been used in [20] for the charge conservation.) In order to get the charge of F-strings in (F, Dp) bound state, we should integrate the above equation on $R^{p-1} \times R^{9-p}$. We have

$$
\begin{aligned}
\int_{R^{p-1} \times R^{9-p}} d\left(e^{-\phi} * G_{3}+\cdots\right) & =\int_{R^{p-1} \times S^{8-p}}\left(e^{-\phi} * G_{3}+\cdots\right), \\
& =\frac{2 \kappa_{0}^{2}}{g T_{f} T_{0}^{p}} \int_{R^{p-1}} * F
\end{aligned}
$$

This gives,

$$
\begin{aligned}
e_{1} & =\frac{1}{\sqrt{2} \kappa_{0}} \int_{R^{p-1} \times S^{8-p}}\left(e^{-\phi} * G_{3}+\cdots\right), \\
& =\frac{\sqrt{2} \kappa_{0}}{g T_{f} T_{0}^{p}} F_{01} A_{p-1}
\end{aligned}
$$

where we have taken $F_{01}$ as the only non-vanishing constant component of the $U(1)$ gauge field strength on the worldvolume of Dp-brane. If we denote $Q_{1}$ as the charge of F-strings per $(2 \pi)^{p-1} \alpha^{\prime(p-1) / 2}$ area, we have

$$
\frac{Q_{1}}{\sqrt{2} \kappa_{0}}=F_{01} / g
$$

where $Q_{1} / \sqrt{2} \kappa_{0}$ is the total tension of these F-strings. So we establish the precise relationship between the F-strings and the electric flux lines. If we take $Q_{1} / \sqrt{2} \kappa_{0}=m T_{f}$ from the above calculated value, we have $F_{01}=g m T_{f}$ which agrees precisely with what we obtained in [5]. Certainly, the other way around is also true.

We now use the metric Eq. (4.5) to calculate the mass per unit p-brane volume for a (F, Dp) bound state and compare the result with what we obtained in [5]. In doing so, we need to generalize the ADM formula in [18] to accommodate the following metric,

$$
d s^{2}=-A(r) d t^{2}+B(r) d r^{2}+r^{2} C(r) d \Omega_{D-p-2}+D(r) \delta_{i j} d x^{i} d x^{j}+E(r) \delta_{k l} d x^{k} d x^{l}
$$

where $D$ is the spacetime dimensions, $p$ is the spatial dimensions of a $p$-brane, and indices $(i, j=1,2, \cdots, m)$ and $(k, l=m+1, \cdots, p)$. If $A(\infty)=B(\infty)=C(\infty)=D(\infty)=E(\infty)=$ $a_{p}$ (with $a_{p}$ a constant) [19], we have the ADM mass per unit $p$-brane volume as 


$$
\begin{gathered}
M_{p}=-\frac{\Omega_{\tilde{d}+1}}{2 \kappa_{0}^{2}}\left[(\tilde{d}+1) r^{\tilde{d}+1} \partial_{r} C(r)+m r^{\tilde{d}+1} \partial_{r} D(r)+(p-m) r^{\tilde{d}+1} \partial_{r} E(r)\right. \\
\left.-(\tilde{d}+1) r^{\tilde{d}}(B(r)-C(r))\right]_{r \rightarrow \infty},
\end{gathered}
$$

with $\tilde{d}=D-p-3$. Applying the above formula to our case, we get the mass per unit p-brane volume for a (F, Dp) bound state as

$$
M_{p}=g \sqrt{n^{2}+g^{2}\left(m-\chi_{B 0} n\right)^{2}} T_{p}^{0}
$$

where we have used $Q_{p}=\Delta_{(m, n)}^{1 / 2} e^{3 \phi_{0} / 2} \sqrt{2} \kappa_{0} Q_{0}^{p} /\left[(7-p) \Omega_{8-p}\right]$, the expressions for $Q_{0}^{p}$ from Eq. (3.12) and the $\Delta$-factor from Eq. (3.5), respectively. Here $T_{0}^{p}=1 /\left[(2 \pi)^{p} \alpha^{(p+1) / 2}\right]$ is the $p$-brane tension units and $g=e^{\phi_{0}}$ is the string coupling. We can relate the $M_{p}$ and the tension $T_{p}(m, n)$ of $(\mathrm{F}, \mathrm{Dp})$ in many ways. For example, they can be related by looking at the scaling behavior of the energy-momentum tensor due to the contribution of the Dpbrane worldvolume action under a constant rescaling of the spacetime metric. But we here use a very simple approach. We know that $M_{p}$ must be proportional to $T_{p}(m, n)$ and the proportionality constant must be some power of the constant $a_{p}$, i.e., $M_{p}=a_{p}^{\alpha} T_{p}(m, n)$ with $\alpha$ an as yet undetermined constant. Note that here $a_{p}=g^{-1 / 2}$. When $m=0, \chi_{B 0}=0$, we know $T_{p}(0, n)=n T_{0}^{p} / g$ and from Eq. (4.17) we also have $M_{p}=g n T_{0}^{p}$. So we have $\alpha=-4$ and we get $M_{p}=g^{2} T_{p}(m, n)$ in general. Using this relation and Eq.(4.14) we find the tension $T_{p}(m, n)$ for the $(\mathrm{F}, \mathrm{Dp})$ bound state as

$$
T_{p}(m, n)=\frac{1}{g} \sqrt{n^{2}+\left(m-\chi_{B 0} n\right)^{2} g^{2}} T_{0}^{p},
$$

which agrees precisely with what we obtained in [0] for $\chi_{B 0}=0$. This also shows that $(\mathrm{F}$, Dp) is a non-threshold bound state.

\section{DECOUPLING LIMIT FOR THE (F, D3) BOUND STATE}

The properties of the (F, Dp) bound states studied in the previous section are either the BPS ones or the consequences of BPS properties. Therefore, it is not surprising that we find agreements in both the spacetime and the worldvolume approaches even though, 
strictly speaking, a flat background is always assumed in the worldvolume study for these bound states in [5]. These calculations clearly demonstrate that our spacetime bound state configurations (F, Dp) are identical to those bound states obtained from the worldvolume study in [5]. The F-strings in (F, Dp) should be identified with the electric flux or field lines in the corresponding gauge theory living on the worldvolume. Now the question is: Can the same $A d S / C F T$ correspondences conjectured by Maldacena in [3] for $p=3$ and by Itzhaki et al in 23] for $p \neq 3$ be proposed in a similar fashion based on the (F, Dp) bound states rather than on the simple Dp branes? If so, can we learn anything new? We will examine these for the $p=3$ case in the following. The analysis for $p \neq 3$ can be made similarly as for the $p=3$ case along the line given in 23 .

In general, when the so-called D-branes appear in string theory, we need to consider not only the modes that propagate in the bulk but also the modes that propagate on the D-branes. The modes propagating on the D-branes are associated with open strings ending on the D-branes. In general, these modes interact not only among themselves but also with the modes propagating in the bulk. However, there exists a limit that decouples the modes propagating on the branes from the modes propagating in the bulk and is also typically a low energy limit. The latter says that the modes on the brane are just the massless ones of the open strings. In this limit, the D-brane theory becomes the corresponding SYM theory (for $p \leq 3)$.

In the case of $p=3$, the D3 brane theory is described by $\mathcal{N}=4 \mathrm{SYM}$ in $1+3$ dimensions under this limit. But the D3 brane configuration is also described in terms of the metric and other fields in the bulk. The so-called decoupling or field theory limit is

$$
g_{\mathrm{YM}}^{2}=2 \pi g=\text { fixed, } \quad \alpha^{\prime} \rightarrow 0,
$$

where $g=e^{\phi_{0}}$ is the string coupling constant and $g_{\mathrm{YM}}$ is the Yang-Mills coupling constant. Note that the ten dimensional Newton constant $\kappa^{2} \sim g^{2} \alpha^{\prime 4}$ vanishes in this limit as it should be. Under this limit, the BPS D3-brane configuration in the bulk becomes flat Minkowski for any fixed non-zero $r$. Therefore, to have something nontrivial, we need to consider an 
additional limit,

$$
U \equiv \frac{r}{\alpha^{\prime}}=\text { fixed }, \quad \alpha^{\prime} \rightarrow 0
$$

The above says that we keep the mass of the stretched strings between D3 branes fixed. This $U$ actually sets the energy scale in the field theory since it is the expectation value of the Higgs. This also implies that we are considering finite energy configurations in the field theory. As discussed in [3], if we consider $n$ parallel D3 branes, then the above decoupling limits say that we are bringing the branes together but the Higgs expectation value corresponding to this separation remains fixed. The resulting theory on the brane is $\mathcal{N}=4 U(n)$ SYM theory in $1+3$ dimensions. In what follows, we simply call Eqs. (5.1) and (5.2) as the decoupling limit.

Under the decoupling limit, the string metric describing the D3 branes becomes $A d S_{5} \times S^{5}$. The isometry of $A d S_{5}$ is $S O(2,4)$ and this is also the conformal group in $1+3$ dimensions. We also have isometries of $S^{5}$ as $S O(6) \sim S U(4)$. This symmetry is identical to the R-symmetry of the $\mathcal{N}=4$ SYM. After including fermionic generators required by supersymmetry, we have the full isometry supergroup $S U(2,2 \mid 4)$ for the $A d S_{5} \times S^{5}$ background, which is identical to the $\mathcal{N}=4$ superconformal group. We would like to emphasize that we have the conformal symmetry $S O(2,4)$ only at $U=0$. For any fixed non-zero $U$, this symmetry is spontaneously broken since $U$ represents the expectation value of the Higgs.

To validate the configuration of D3 branes as a stringy one, the large $n$ limit has to be taken given the fixed but small string coupling strong-weak duality symmetry in both type IIB supergravity and $\mathcal{N}=4$ SYM. These symmetry identifications, among other things, led Maldacena to conjecture that the large $n, \mathcal{N}=4$ SYM theory with gauge group $U(n)$ is actually equivalent to the ten dimensional type IIB supergravity on $A d S_{5} \times S^{5}$. However, the supergravity itself is not a consistent quantum theory. On the other hand, the SYM is a unitary quantum theory. Moreover,

\footnotetext{
${ }^{15}$ For large $g$, the D-string frame is chosen.
} 
the above symmetry identifications are independent of the large $n$ limit (even though it is needed to validate the solution). These two facts led Maldacena further to conjecture that type IIB string on $A d S_{5} \times S^{5}$ is equivalent to the $\mathcal{N}=4$ SYM theory for a general $n$.

Let us now apply the same process to the (F, D3) configuration obtained in section 3, i.e., taking the decoupling limit given by Eqs. (5.1) and (5.2). In other words, we should examine the near-horizon geometry of $(F, D 3)$ configuration with the string coupling $g$ fixed $\square$. The near horizon string-frame metric in this case is

$$
\begin{aligned}
d s^{2}= & \alpha^{\prime}\left[\frac{U^{2}}{\sqrt{4 \pi n g}}\left(\frac{n^{2} e^{-\phi_{0}}}{\Delta_{(m, n)}}\right)^{3 / 4}\left(-\left(d x^{0}\right)^{2}+\left(d x^{1}\right)^{2}\right)\right. \\
& +\frac{U^{2}}{\sqrt{4 \pi n g}}\left(\frac{n^{2} e^{-\phi_{0}}}{\Delta_{(m, n)}}\right)^{-1 / 4}\left(\left(d x^{2}\right)^{2}+\left(d x^{3}\right)^{2}\right) \\
& \left.+\sqrt{4 \pi n g}\left(\frac{n^{2} e^{-\phi_{0}}}{\Delta_{(m, n)}}\right)^{1 / 4}\left(\frac{d U^{2}}{U^{2}}+d \Omega_{5}^{2}\right)\right]
\end{aligned}
$$

and the dilaton is

$$
e^{\phi}=\left(n^{2} e^{-\phi_{0}} / \Delta_{(m, n)}\right)^{1 / 2} e^{\phi_{0}}
$$

It is clear from (5.3) and (5.4) that unlike the simple D3 brane, the near-horizon geometry in this case is not automatically $A d S_{5} \times S^{5}$ and the effective string coupling, $e^{\phi}$, is still a finite constant but is less than the string coupling $g$.

The above features may be expected given the appearance of an infinite number of Fstrings in (F, D3) and the non-threshold bound state nature of this configuration. On the other hand, in the decoupling limit, we expect that D3 brane can be described equivalently either by the SYM theory on the brane or by the type IIB supergravity (or string) in the bulk. Depending on whether we consider excitations with respect to the simple D3-brane

\footnotetext{
${ }^{16}$ For simplicity, we set $\chi_{B 0}=0$ from now on.

${ }^{17}$ The dilaton for (F, D3) is actually not a constant in general, nevertheless it is bounded as $\left(n^{2} e^{-\phi_{0}} / \Delta_{(m, n)}\right)^{1 / 2} e^{\phi_{0}}<e^{\phi}<e^{\phi_{0}}$, where the upper bound is obtained at $r=\infty$ and the lower bound at $r=0$.
} 
vacuum or the (F, D3) bound state (chosen as a new vacuum), we have two descriptions which are different in appearance but probably equivalent in essence. Let us first discuss the possible underlying IIB string/SYM correspondence for each of the above descriptions in the strong sense, i.e., not taking the large $n$ limit. We will discuss the large $n$ limit in the end.

If we choose the simple BPS D3 brane configuration in the bulk or equivalently no excitations in the SYM on the brane as the vacuum, then the F-strings in the bulk or equivalently the electric flux lines in the SYM should be formed due to excitations with respect to this vacuum. We know that the energy per unit 3-brane volume associated with the F-strings in the bulk or the energy density associated with the electric flux lines on the brane is finite. This implies that we have an infinite amount of energy associated with either the F-strings or the electric flux lines. In other words, we should have $U=$ $\infty$, which implies that the original conformal symmetry group $S O(2,4)$ must be broken spontaneously to some smaller symmetry group consisting of at most some translational and rotational symmetries. It is not difficult to figure out the origin of $U=\infty$ if we recall the method we used in [5] to find the (F, D3) bound state. As discussed in [5], the charge conservation must imply that the infinite parallel F-strings in (F, D3) originate from Fstrings along the radial coordinate $r$ ending on the $x^{2} x^{3}$-plane placed at $x^{1}=-\infty$ \&. These F-strings ending on $x^{2} x^{3}$ plane are the open strings, each semi-infinitely long, giving $U=\infty$. Inside the bulk, we have F-strings along $x^{1}$-axis, therefore we expect an $S O(1,1)$ symmetry. Because of the ending or the charges on $x^{2} x^{3}$-plane placed at $x^{1}=-\infty$, we expect that $S O(2,4) \rightarrow S O(1,1) \times S O(2)$ for this near-horizon geometry. Thus we find that we actually have $S O(1,1) \times S O(2) \times S O(6)$ isometries for the near-horizon geometry of the (F, D3) bound state for the choice of vacuum. On the SYM side, we have the same answer. Dilation and the special conformal transformations are broken spontaneously for the same reason

\footnotetext{
${ }^{18}$ This is chosen in order to preserve the $\mathrm{SO}(6)$ isometry of $S^{5}$.
} 
mentioned above. Only the subgroup $S O(1,1) \times S O(2)$ of $S O(2,4)$ will leave $F_{01}=g m T_{f}$ invariant. So, following the similar argument as for the $A d S_{5} / C F T_{4}$ correspondence, we may suggest that the type IIB string on this geometry is equivalent to the $\mathcal{N}=4 \mathrm{SYM}$ theory in a state with quantized electric flux lines.

Let us investigate how the (F, D3) bound state emerges as our vacuum for the second description. Examining carefully the metric and the dilaton, we can see that $n^{2} e^{-\phi_{0}} / \Delta_{(m, n)}$ factor is the source which causes the near-horizon geometry to be not automatically $A d S_{5} \times S^{5}$ and the effective string coupling to be less than the string coupling $g$. Explicitly, we have this factor

$$
\frac{n^{2} e^{-\phi_{0}}}{\Delta_{(m, n)}}=\left(1+\left(\frac{g m}{n}\right)^{2}\right)^{-1}
$$

Now if we rescale the coordinate time $x^{0}$ and the coordinate $x^{1}$ by a factor $\left(n^{2} e^{-\phi_{0}} / \Delta_{(m, n)}\right)^{-1 / 2}$, then in terms of the new coordinates the near-horizon geometry is $A d S_{5} \times S^{5}$. So, if we rewrite the metric in terms of the effective string coupling

$$
g_{\mathrm{eff}} \equiv e^{\phi}=\frac{g}{\left(1+\left(\frac{g m}{n}\right)^{2}\right)^{1 / 2}}
$$

we have

$$
d s^{2}=\alpha^{\prime}\left[\frac{U^{2}}{\sqrt{4 \pi n g_{\mathrm{eff}}}}\left(-\left(d x^{0}\right)^{2}+\left(d x^{1}\right)^{2}+\left(d x^{2}\right)^{2}+\left(d x^{3}\right)^{2}\right)+\sqrt{4 \pi n g_{\mathrm{eff}}}\left(\frac{d U^{2}}{U^{2}}+d \Omega_{5}^{2}\right)\right]
$$

The radius for the $A d S_{5}$ and the $S^{5}$ is now given as

$$
R^{2} / \alpha^{\prime}=\sqrt{4 \pi n g_{\mathrm{eff}}}
$$

The information about the F-strings in the (F, D3) bound state disappears in the above metric. Since the resulting near-horizon geometry differs from that of a simple D3 brane with 5-form flux $n$ only in the string coupling, we expect that the effects of the F-strings are encoded in the effective string coupling $g_{\text {eff }}$. We will see that this is indeed true. With respect to the new coordinates, we have $S O(2,4) \times S O(6)$ isometries in the bulk at least 
in appearance. How can we reconcile this with what we just discussed above on the SYM side? Moreover, what is the physics behind such a rescaling?

In the case of simple D3 branes, the string coupling $g=e^{\phi_{0}}$ remains the same whether we are at $r=\infty$ or at $r=0$. This fact enables us to fix the gauge coupling as $g_{\mathrm{YM}}^{2}=2 \pi g$ for the SYM theory describing the D3 branes in the decoupling limit. We do not expect that such a relation will be modified for any finite energy excitations $[$. However, the story here is different. The gauge coupling should, in the present case, be related to $g_{\text {eff }}$ rather than $g$ as $g_{\mathrm{YM}}^{2}=2 \pi g_{\text {eff }}$.

With respect to the metric in Eq. (5.7), we may say that a simple D3 brane with 5-form flux $n$ plays as an "effective" vacuum configuration in the bulk with the string coupling $g_{\mathrm{eff}}$. This simple D3 brane is also described by a $\mathcal{N}=4 U(n)$ SYM theory in $1+3$ dimensions with gauge coupling $g_{\mathrm{YM}}^{2}=2 \pi g_{\mathrm{eff}}$. This is just the usual $A d S_{5} / C F T_{4}$ correspondence except we have a new coupling constant. Let us dig out the mystery behind the above "effective" vacuum. We expect that the tension for the simple D3 brane is $n T_{0}^{3} / g_{\text {eff }}$. If we use the explicit expression for $g_{\text {eff }}$ from Eq. (5.6), this tension is

$$
\frac{n T_{0}^{3}}{g_{\mathrm{eff}}}=\frac{T_{0}^{3}}{g} \sqrt{n^{2}+g^{2} m^{2}},
$$

which is just the tension for the (F, D3) bound state. So this "effective" vacuum is nothing but the (F, D3) bound state. As anticipated, the effects of the F-strings in (F, D3) is encoded into the string coupling constant.

On the SYM side, we have already hinted that the gauge coupling is now given by $g_{\mathrm{YM}}^{2}=2 \pi g_{\mathrm{eff}}$. In the linear approximation,

$$
\frac{1}{g_{\mathrm{eff}}}=\frac{1}{g}+\frac{1}{2 g}\left(\frac{g m}{n}\right)^{2},
$$

${ }^{19}$ But it could be modified if the energy involved is infinite, for example, the case we are presently studying. If this happens, the SYM theory may no longer be valid in describing the underlying physics in general and string theory should be used instead. However, our discussion above for the first description may still be valid since it is based on the BPS (F, D3) configuration. 
where the second term for $n=1$ is nothing but the contribution from the energy density of the electric flux lines. In other words, the effect of the energy of the quantized electric flux lines is absorbed into the gauge coupling. This is in accordance with our spacetime picture just discussed. Therefore, the resulting SYM theory should be the one describing the excitations with respect to the (F, D3) bound state. The superconformal symmetry should then be restored. We therefore have a consistent picture both in the bulk and on the brane in the decoupling limit.

Our study lends further support for Maldacena's $A d S_{5} / C F T_{4}$ correspondence and shows that it holds true even for the non-trivial D3 brane configurations. The new input for this correspondence is that we should use the effective string coupling constant instead of the usual one.

The two descriptions studied may be equivalent but the picture for the first one is surely complicated. The reason for this is our improper choice of the vacuum state. If we take the large $n$ limit, i.e., $n \gg m$ for fixed $g$, the effective string coupling becomes the usual string coupling $g$. The F-strings in the (F, D3) now play a minor role with respect to the D3 branes which can be seen from the tension formula $T_{3}(m, n)=n / g\left[1+(m / n)^{2} g^{2} / 2\right] T_{0}^{3}$. On the SYM side, on the other hand, we have the gauge coupling $g_{\mathrm{YM}}^{2}=2 \pi g\left(1-(m / n)^{2} g^{2} / 2\right)$ which implies that electric flux lines plays the minor role. Thus the $A d S_{5} / C F T_{4}$ correspondence with respect to $(\mathrm{F}, \mathrm{D} 3)$ bound state is simply reduced to that of the simple D3 brane configuration. We also expect that the full isometry supergroup $S U(2,2 \mid 4)$ in the bulk and the superconformal symmetry in the SYM theory are restored under this limit in our first description above. This description is therefore also reduced to the usual $A d S_{5} / C F T_{4}$ correspondence. This large $n$ limit also validates the (F, D3) configuration as a stringy one.

Let us now comment on the effective string coupling $g_{\text {eff }}$ given by Eq. (5.6) in the nearhorizon region. It is clearly quantized in terms of relatively prime integers $n$ and $m$ and is always less than the string coupling $g$. In particular, in the limit $m \gg n$, it becomes $g_{\text {eff }}=n / m \ll 1$, independent of the string coupling $g$. In other words, the effective string coupling in the near-horizon region is completely determined by integer $n$, the 3 -brane 
charge, and $m$, the number of F-strings per $(2 \pi)^{2} \alpha^{\prime}$ area over the $x^{2} x^{3}$-plane, in this limit.

We will continue this study in a more general D3-brane configuration, namely, ((F, D1), D3) bound state in a forthcoming paper [24].

\section{CONCLUSION}

To summarize, we have constructed, in this paper, explicitly the (F, Dp) non-threshold bound state configurations for $2 \leq p \leq 7$, starting from the known (F, D1) configuration of Schwarz by T-duality transformation along the transverse directions of the strings. We have also presented the solutions for (W, Dp) non-threshold bound state configurations for

$0 \leq p \leq 6$ by T-duality transformations on the newly obtained (F, Dp) ones along the longitudinal direction of F-strings in (F, Dp). We have shown explicitly that there are $m$ F-strings per $(2 \pi)^{p-1} \alpha^{\prime(p-1) / 2}$ of $(p-1)$-dimensional area which agrees with our previous result based on the worldvolume study. We have also calculated the tensions for the (F, Dp) bound states which once again agree with our previous results. These two facts confirm our assertion made in our earlier paper that the bound state studied in [5] can indeed be identified with the (F, Dp) bound states here. All of these bound states preserve one half of the spacetime supersymmetries.

From the D-brane worldvolume point of view, each of these bound states consists of a Dp-brane carrying certain units of quantized electric flux or field line. But from the spacetime point of view, we have type II strings (either type IIA or IIB strings) lying along one direction in the corresponding Dp-brane worldvolume. In this paper, we have shown that for each of the bound states considered, the type II strings can be identified with the electric flux lines in the gauge theory living on the corresponding Dp brane worldvolume. In the $p=3$ case, we have studied the corresponding decoupling limit and found that the $A d S_{5} / \mathrm{CFT}_{4}$ correspondence may still hold true but now with respect to the (F, D3) bound state with an effective string coupling constant. The F-strings in the (F, D3) bound state or the electric flux lines in the corresponding SYM theory play the role of reducing the 
respective coupling constant in general. The string coupling in the near-horizon region is found to be quantized in terms of the relatively prime integers $n$, the 3-brane charge, and $m$, the number of F-strings per $(2 \pi)^{2} \alpha^{\prime}$ area over the $x^{2} x^{3}$-plane. It becomes independent of the asymptotic string coupling $g$ in the limit $m \gg n$.

\section{ACKNOWLEDGMENTS}

We would like to thank Mike Duff for reading the manuscript and Chris Pope for discussions. JXL acknowledges the support of NSF Grant PHY-9722090. 


\section{REFERENCES}

[1] J. Schwarz, Phys. Lett. B360 (1995) 13 (see hep-th/9508143 for the most recent revision of this paper).

[2] C. Callan and J. Maldacena, Nucl. Phys. B513 (1998) 198.

[3] J. Maldacena, Adv. Theor. Math. Phys. 2 (1998) 231.

[4] J. Polchinski, Phys. Rev. Lett. 75 (1995) 4724.

[5] J. X. Lu and S. Roy, "(m,n)-String-Like Dp-Brane Bound States", hep-th/9904112.

[6] H. Arfaei and M. M. Sheikh Jabbari, Nucl. Phys. B526 (1998) 278.

[7] J. G. Russo and A. A. Tseytlin, Nucl. Phys. B490 (1997) 121.

[8] M.B. Green, N. D. Lambert, G. Papadopoulos and P.K. Townsend, Phys. Lett. B384 (1996) 86 .

[9] M. Costa and G. Papadopoulos, Nucl. Phys. B510 (1998) 217.

[10] J. Polchinski, String Theory, Vol.II (Cambridge University Press, 1998), J. Izquierdo, N. Lambert, G. Papadopoulos and P. K. Townsend, Nucl. Phys. B460 (1996) 560, M. Green and M. Gutperle, Nucl. Phys. B476 (1996) 484, G. Lifschytz, Nucl. Phys. B499 (1997) 283, J. Breckenridge, G. Michaud and R. Myers, Phys. Rev. D55 (1997) 6438.

[11] E. Bergshoeff, E. Eyras, R. Halbersma, J. P. van der Schaar, C. M. Hull and Y. Lozano, "Space-time Filling Branes and Strings with Sixteen Supercharges", hep-th/9812224.

[12] A. Dabholkar, J. P. Gauntlett, J. A. Harvey and D. Waldram, Nucl. Phys. B474 (1996) 85.

[13] H. Lu, C. N. Pope and K. S. Stelle, Nucl. Phys. B481 (1996) 313.

[14] M. J. Duff, H. Lu and C. N. Pope, Nucl. Phys. B532 (1998) 181.

[15] M. J. Duff, P. S. Howe, T. Inami and K. S. Stelle, Phys. Lett. B191 (1987) 70. 
[16] I. V. Lavrinenko, H. Lu, C. Pope and K. Stelle, "Superdualities, Brane Tensions and Massive IIA/IIB Duality", hep-th/9903057.

[17] E. Bergshoeff, C. Hull and T. Ortin, Nucl. Phys. B451 (1995) 547.

[18] M. J. Duff, R. R. Khuri and J. X. Lu, Phys. Rept. 259 (1995) 213.

[19] T. H. Buscher, Phys. Lett. B194 (1987) 59.

[20] A. Strominger, Phys. Lett. B383 (1996) 44.

[21] J. X. Lu, Phys. Lett. B313 (1993) 29.

[22] Since Ricci tensor is inert under an overall constant rescaling of the metric, it will not change the total energy-momentum pseudotensor. Therefore, such a rescaling of metric will not change the mass per unit coordinate $p$-brane volume (In the present case, this coordinate volume is also the $p$-brane volume measured in string metric at the spatial infinity of the transverse space.).

[23] N. Itzhaki, J. M. Maldacena, J. Sonnenschein and S. Yankielowicz, Phys. Rev. D58 (1998) 46004.

[24] J. X. Lu and S. Roy, "((F, D1), D3) Bound State and Its T-Dual Daughters" hepth/9905014. 